\title{
Tetrachromacy: The mysterious case of extra-ordinary color vision
}

Gabriele Jordan and John Mollon

Recent years have witnessed a growing public interest in human tetrachromacythe possibility that a subpopulation of women enjoy an extra dimension of color vision. Yet, by contrast, rigorous studies of this unusual phenotype are sparse. The aim here is to offer the reader a guide to the facts and myths regarding this potential 'superpower' and to address the core methodological issues that need to be considered when investigating it.

\section{Introduction}

Tetrachromatic color vision allows an individual to discriminate colors along a perceptual dimension that is unavailable to the normal person. Most people's color perception is 'trichromatic', i.e. their retinas contain three types of light-sensitive cone photopigment, absorbing maximally in the long- (L), middle- $(\mathrm{M})$ and shortwavelength (S) parts of the spectrum. Behaviorally, in a color-matching experiment using spectral lights, they can match any color with just three variable colors: A match between two stimulus fields can be achieved simply by equating the triplets of cone signals in the two fields [1]. For tetrachromacy to arise, there must be four distinct types of cone photopigment in the retina and their four independent signals or three independent ratios - must be available to later stages of processing.

One reason that tetrachromacy is of interest lies in its implications for plasticity within the visual system: It is implausible that an additional chromatic pathway has evolved to benefit a small minority of our conspecifics who are heterozygous for X-linked cone photopigments. But how then do the retina and the cortex take advantage of an additional, independent cone signal? Even with four types of cones, vision might remain trichromatic in the sense that only three variables are needed in a colormatching experiment [2]. We have called this 'weak tetrachromacy' [3]. The more interesting case is 'strong tetrachromacy', where a woman requires four independent stimuli in matching colors, and it is this possibility we discuss below. 
In this review we address the following questions: (1) How can tetrachromacy arise and why only in women? (2) What combinations of cone photopigments are required for tetrachromacy? (3) What are the retinal mechanisms allowing for plasticity of the visual system? (4) What are the methodological challenges to demonstrating the existence of this phenotype? (5) Does tetrachromacy confer any advantages?

\section{How can tetrachromacy arise and why only in women?}

The photopigments ('opsins') in the L and M cones are encoded by adjacent genes on the X-chromosome [4*]. Men inherit a single, maternal X-chromosome, whereas women inherit one from each parent. However, to compensate for the difference in X-chromosome dosage between men $(X Y)$ and women $(X X)$, most genes on one $X$ chromosome are inactivated in any given cell of a woman's body [5*]. This inactivation is random and initiated very early in embryonic development. Consider now a woman whose $\mathrm{X}$-chromosomes carry different versions (alleles) of, say, the $\mathrm{M}$ opsin gene. If these alleles encode photopigments that differ in their wavelengths of maximal sensitivity, then her retina will contain four types of cone photopigment: $S$, $M, L^{\prime}$ (or shifted M), and L [Fig 1a]. X-chromosome inactivation segregates the alternative gene products in different cones and so provides a retinal cone mosaic with four cone types [Fig 1b]. The size of retinal cone patches will depend on the time of onset of $X$ inactivation and the migration of cones in the developing retina.

The existence of tetrachromatic women was first postulated by de Vries [6]: The women that he thought "must be tetrachromatic" were the daughters of anomalous trichromats - for whom one photopigment ( $M$ or $L)$ is spectrally shifted $\left[7^{*}\right]$. His hypothesis gained plausibility from the discovery in Platyrrhines - New World monkeys - of a remarkable polymorphism of cone photopigments [4*,8]. Most species of Platyrrhini are basically dichromatic, combining an S-cone photopigment with one that peaks in the middle- to long-wavelength spectral region; but there are typically three (or more) X-chromosome alleles for the latter pigment. Males (XY) must always be dichromatic, but a female $(X X)$ will be trichromatic if she inherits two different versions of the middle-/long-wave gene. If random X-chromosome inactivation can give rise to trichromats in a basically dichromatic species, could it not yield tetrachromacy in ours? 


\section{Which complement of photopigments gives rise to tetrachromacy? Two alternative hypotheses.}

In our species, the genes for color vision are highly polymorphic, and probably most women are heterozygous in the sense that the arrays of opsin genes on their two $X$ chromosomes are not identical. But how many women are potentially tetrachromatic?

One of the most commonly observed polymorphisms is that at site 180 in the aminoacid sequence of the $L$ cone opsin. In $62 \%$ of Caucasians serine (ser) is present at this site, but in $38 \%$, alanine (ala) [9]. The two versions of $L$ photopigments differ by $4-5 \mathrm{~nm}$ in their wavelength of peak sensitivity, with ser180 being more sensitive to longer wavelengths $[10,11]$. When this ser180ala polymorphism was first described, one of us raised the possibility that women heterozygous at site 180 might be tetrachromatic [12]; and in a series of subsequent papers, Jameson and colleagues have suggested that this polymorphism may indeed underlie tetrachromacy [13-15]. Tetrachromacy could then be very common: the proportion of ser180ala heterozygotes in a Caucasian population would be $1-\left(0.62^{2}+0.38^{2}\right)$, i.e. about $47 \%$. The latter estimate has been confirmed in a gene expression study of nine female donor eyes [16].

Would a separation of $4-5 \mathrm{~nm}$ between two $L$ photopigments be enough to give the potential tetrachromat a salient, extra signal? Here we can draw on earlier modelling of 'anomalous trichromacy' - the mild colour vision deficiency seen in $\sim 6 \%$ of men, where either the normal $\mathrm{L}$ cone or the normal $\mathrm{M}$ cone is replaced by a cone shifted in spectral position $\left[7^{*}\right]$. If the two remaining cones in the middle-/long-wave region are separated by only $4-5 \mathrm{~nm}$, the estimated number of discriminable colors in realworld hyperspectral images would increase relative to the number discriminated by a dichromat who had only L and S cones [17]; but performance would be poorer than that of a normal trichromat - or that of an anomalous trichromat with more widely separated photopigments.

Since the common polymorphism at amino acid site 180 would give only a small spectral separation, and since in practice strong tetrachromacy appears to be rare, 
we ourselves have favored an alternative hypothesis, that tetrachromacy has the best chance of arising in those carriers of anomalous trichromacy who have normal $L$ and $\mathrm{M}$ photopigments plus an anomalous photopigment that lies midway between them. Anomalous photopigments of this type are thought to arise from hybrid genes. The highly homologous [18] $\mathrm{L}$ and $\mathrm{M}$ genes may misalign at meiosis. If now a crossing over occurs within the mispaired genes, the recombined outcome may consist partly of the normal $L$ sequence and partly of the normal $M$ sequence. The expressed opsin typically has a peak sensitivity intermediate between $L$ and $M$. We note that the strongest candidate for tetrachromacy identified so far (cDa29) [19] has an estimated separation of $12 \mathrm{~nm}$ between her anomalous, L' cones and her L cones.

Anomalous trichromats vary widely in their ability to discriminate in the red-green spectral range $\left[7^{*}\right]$ : Some are nearly as limited as dichromats, while a tiny minority the 'Minimalanomale Trichromaten' of Vierling [20] - perform normally on standard tests for color deficiency and have matching ranges close to zero on the anomaloscope. Our hypothesis is that it is the daughters of the latter men who are most likely to become tetrachromatic.

\section{Post-receptoral mechanisms allowing for tetrachromatic signals}

In order to perceive colors, and to discriminate between them, the cone activations from different cone types must be compared. Two independent, post-receptoral channels have been identified for this task [21-24]. Of particular interest for tetrachromatic vision is the phylogenetically younger, 'red-green' opponent channel. Midget ganglion cells of the parvocellular pathway have receptive fields that are divided into spatially and chromatically antagonistic centers and surrounds. In the fovea, their center input is drawn from a single cone of one type, whereas the surround input is likely to be drawn indiscriminately from $L$ and $M$ cones (mixed surround) [22]. Such a channel lends itself to tetrachromatic vision as its chromatic specificity is determined primarily by the type of cone feeding into the center [8]. Thus, if the signal from a single L' cone is compared to the pooled input of other cones, say $L+L^{\prime}$ in the case of men with deuteranomaly (Da), a female carrier (cDa) will have the neural basis for similar comparisons. Figure $1 \mathrm{~b}$ shows a schematic 
representation of a tetrachromatic cone mosaic with a deuteranomalous ( $L^{\prime}$ vs $\left.\left(L^{\prime}+L^{\prime}\right)\right)$ as well as normal $(L$ vs $(L+M)$ and $M$ vs $(L+M))$ comparisons by three potential midget ganglion cells. If all ratios of cone excitations are capable of providing salient input and are preserved in cortical mechanisms, then strong tetrachromacy should result. The chromatic signal should be largest if the cone input into the surround of the receptive field is relatively unmixed. This is more likely to occur in cases of cone clumping and/or cone mosaics that are biased towards L-type cones [25]. Note, that such retinas will yield spectral luminous efficiency functions (which depend on the summed signal from L- and M-type cones) very different from normal. Thus it is especially important to eliminate or adjust for luminance cues when testing for tetrachromacy (see below). cDA29's relative sensitivities to the red and green phosphors of a Sony Trinitron monitor [25] were indicative of a retinal mosaic dominated by L-type cones [19].

\section{Methodological considerations}

Knowledge of an individual's receptor types, either through molecular genetic techniques or through classifying a carrier's first-degree relative(s), is not sufficient to indicate the dimensionality of her color vision $\left[19,26^{\star}\right]$. How can tetrachromacy be experimentally revealed?

Color matching using spectral lights: No ordinary RGB color display is suitable for such a test. Jordan and Mollon [3] used a specifically designed colorimeter to test whether obligate carriers make unique color matches in the 546 to $690 \mathrm{~nm}$ spectral range compared to control participants when given an additional primary in a ratiomatching task. Only one (cDa1) out of 14 obligate carriers for simple anomalous trichromacy made such unique matches.

Color discrimination using spectral lights: We later argued that a forced-choice discrimination task would be a more objective test for tetrachromacy than a criterionbased matching task [19]. As before, the primaries were in the spectral range where $S$ cone are insensitive. A triplet of three successive lights was presented. One light was a red-green mixture $(546+670 \mathrm{~nm})$ that could vary in the proportion of red, and the other two were a monochromatic yellow (590 nm). Observers had to identify the 
red-green mixture. Since all normal trichromats can make a dichromatic color match in this reduced spectral range, they were expected to fail to discriminate for some red-green mixture and some intensity of the monochromatic yellow. In contrast, a strong tetrachromat was predicted to always detect the mixture. Only one (cDa29) out of $18 \mathrm{cDa}$ and none of $7 \mathrm{cPa}$ participants performed according to the prediction. All control subjects failed to discriminate. Since cDa29 could not make a match with only two primaries available in the Rayleigh region, she was classified as a strong tetrachromat.

Color discrimination using surface colors: A good test for strong tetrachromacy is to target carriers of deuteranomaly with a test specifically designed to reveal the anomalous L vs ( $\left.L+L^{\prime}\right)$ channel [27]. Deuteranomalous men with small Rayleigh matching ranges (good discrimination ability) are able to make discriminations not achievable by normal observers $[19,27,28]$. If the $L$ vs $\left(L+L^{\prime}\right)$ channel is salient enough in $\mathrm{CDa}$ individuals, they ought to be able to make the same discriminations. Luminance was jittered across the normal and deuteranomalous dimensions to ensure that discriminations were based on chromaticities. Multidimensional scaling (MDS) of each observer's dissimilarity ratings was used to reconstruct their subjective color space. Four out of nine cDa observers were found to be able to make discriminations on this test and their MDS solution correlated significantly with the theoretical $L$ vs $\left(L+L^{\prime}\right)$ channel. They included cDa29 [19].

Other approaches: To "extend the stimulus complexity beyond the [reductionist] color-matching configuration" [13, p247] and to improve the ecological validity of the experimental tests for tetrachromacy, Jameson and colleagues have introduced two different approaches.

First, they asked observers to delineate the perceived chromatic bands of a projected diffraction spectrum by drawing lines on tracing paper [13]. They report a richer color experience in 23 carriers of the $\mathrm{L}$ cone ser180ala genotype compared to controls. On average, these carriers delineated 10 (range 6-15.67) spectral bands compared to 7.6 (range 5.67-11.83) and 7.3 by normal females and males respectively. Intriguingly, those carriers who indicated the highest number if delineations, showed a reduced gamut (i.e. the endpoints of the spectrum) of 
perceived colors compared to other participants. Smeulders et al [29] using the same approach, report that in normal trichromats increasing the number of delineations in the projected spectrum increases the perceived chromatic bands from 7 to 25-30. This dramatic increase was explained as a by-product of an increase in luminance contrast transients introduced by the lines made. We note that the bands of a projected spectrum are not of equal luminance [29] and that carriers will have altered sensitivities to given spectral bands owing to their altered luminous efficiency function.

Second, the authors have linked the ser180ala genotype to the experiential sensations of selected carriers' and to the ability to communicate these sensations [14]. In particular they have asked whether tetrachromacy reveals itself in the use of color in artistic expression. Two out of four carriers were professional artists, and one of two normal female controls was also an artist. The minimum motion technique of Anstis and Cavanagh [30] was used to estimate the subjective isoluminance points of 20 colors (produced on a RGB monitor) to four levels of gray (of unspecified luminance) and one uniform background color. Results for one of two potential tetrachromatic artists (subject CA) show different equiluminant settings for some of the color patches compared to other observers. It was also found that her artwork, that aims to represent what she sees, indeed differs in color use from that of the trichromatic artist. CA's sister's (LA) artwork also uses color to a great effect.

Recruitment issues and population statistic. Behavioral studies rely on the participation of volunteers, but the recruitment strategy may create biases that can influence inferences from the study's outcome. For example, if one recruits mothers who have color-deficient sons [3], the sons tend to be more severely affected as they are more aware of their deficiency. Necessarily, one misses the minimal anomalous trichromats [20] who are likely to have the largest spectral separation between their $X$-linked cone photopigments and this might partly explain the low number of potential tetrachromats found. Using the 2012 UK Census data, we estimated a frequency of 3.9 million carriers of hybrid genes, but only 48,585 carriers who are expected to have the optimal spectral separation of $12 \mathrm{~nm}$ between their L/L' (M/M') photopigments. Population screening should therefore be all inclusive and performed with an anomaloscope or other appropriate screening test. 


\section{What are the potential benefits to a tetrachromat?}

We do not yet know whether functional tetrachromacy confers any advantages, but it seems clear that the benefits, if there are any, are subtle. On the basis of anecdotal evidence we have speculated that there may be signals in the skin tone of conspecifics that are linked to health or disease [31]. In such cases early detection will be advantageous.

Will a potential tetrachromat be a more successful artist? If she uses her color palette veridically to render her sensations, then it is not clear how she can communicate the added richness of her private gamut to those who live in an impoverished perceptual world.

\section{Conclusion}

Tetrachromacy, a phenotype arising from X-linked genotypic variants, is an exciting prospect and it now seems likely that there are individuals who fit the expected profile. However, the relationship between the existence of a fourth class of retinal cone and the dimensionality of colour vision is more complex than previously thought. A number of factors may determine whether functional tetrachromacy arises, such as the spectral distance between the peak sensitivities of the cones, the distribution and relative number of cone types across the retina, and the relative strength or weighting of chromatic signals that are sent from the retina to the cortex. Taking into account the sub-optimal recruiting strategies for experimental studies and the lack of an appropriate screening tool to provide a population statistic, there are still more questions than answers.

\section{Conflict of interest statement}

Nothing declared

\section{References and recommended reading \\ * of special interest}


1. Stockman A, Brainard DH: Fundamentals of color vision I: color processing in the eye. In Handbook of Color Psychology. Edited by Elliot AJ, Fairchild MD, Franklin, A. Cambridge: Cambridge University Press; 2015: 27-69

2. Nagy AL, MacLeod DIA, Heyneman NE, Eisner A: Four cone pigments in women heterozygous for color deficiency. J Opt Soc Am 1981, 71: 719-722

3. Jordan G, Mollon JD: A study of women heterozygous for colour deficiencies. Vision Res 1993, 33: 1495-1508

*4. Carvalho LS, Pessoa DMA, Mountford JK, Davies WIL, Hunt DM: The Genetic and Evolutionary Drives behind Primate Color Vision. Front Ecol Evol 2017, 5: 34: $1-11$

A recent review discussing the different pathways for trichromacy in Old World and New World primates.

*5. Balaton BP, Dixon-McDougall T, Peeters SB, Brown CJ: The exceptional nature of the X chromosome. Hum Mol Genet 2018, 27: R242-R249

A review of $\mathrm{X}$-chromosome inactivation and of its exceptions.

6. De Vries $\mathrm{H}$ : The fundamental response curves of normal and dichromatic and trichromatic eyes. Physica 1948, 14: 367-380

*7. Bosten J: The known unknowns of anomalous trichromacy. Curr Op Beh Sci in preparation 2019

A discussion, in this issue, of inter-individual differences in anomalous trichromacy covering genetics, phenotypic variability and modelling.

8. Mollon JD, Bowmaker JK, Jacobs GH: Variations of colour vision in a New World primate can be explained by polymorphism of retinal photopigments. Proc Roy Soc London B 1984, 222: 373-399

9. Winderickx J, Lindsey DT, Sanocki E, Teller DY, Motulsky AG, Deeb SS:

Polymorphism in red photopigment underlies variation in colour matching. Nature 1992, 356: 431-433

10. Neitz M, Neitz J, Jacobs, GH: Spectral tuning of pigments underlying redgreen color vision. Science 1991, 252: 971-974

11. Merbs SL, Nathans J: Absorption spectra of the hybrid pigments responsible for anomalous color vision. Science 1992, 258: 464-466

12. Mollon JD: Worlds of difference. Nature 1992, 356: 378-379 
13. Jameson KA, Highnote SM, Wasserman LM: Richer color experience in observers with multiple photopigment opsin genes. Psychon Bull Rev 2001, 8:244-261

14. Jameson KA, Winkler AD, Goldfarb K: Art, interpersonal comparisons of color experience, and potential tetrachromacy. In International Symposium on Electronic Imaging, Human Vision and Electronic Imaging 145, Society for Imaging Science and Technology, 2016:1-12

15. Bochko VA, Jameson KA: Investigating potential human tetrachromacy in individuals with tetrachromat genotypes using multispectral techniques. In International Symposium on Electronic Imaging, Human Vision and Electronic Imaging 542, Society for Imaging Science and Technology, 2018:1-12

16. Neitz M, Kraft TW, Neitz J: Expression of L cone pigment gene subtypes in females. Vision Res 1998, 38: 3221-3225

17. Thomas PB, Formankiewicz MA, Mollon JD: The effect of photopigment optical density on the color vision of the anomalous trichromat. Vision Res 2011, 51: 2224-2233

18. Zhao Z, Hewett-Emmett D, Wen-Hsiung L: Frequent gene conversion between human red and green opsin genes. J Mol Evol 1998, 46: 494-496

19. Jordan G, Deeb SS, Bosten JM, Mollon JD: The dimensionality of color vision in carriers of anomalous trichromacy. J Vis 2010, 10:12

20. Vierling O: Die Farbensinnprüfung bei der Deutschen Reichsbahn.

Bernecker: Melsungen;1935

21. Mollon JD: "Tho' she kneeled in that place where they grew..." The uses and origins of primate color vision. J exp Biol 1989, 146: 21-38

22. Dacey DM: Primate retina: cell types, circuits and color opponency. Prog. Ret Eye Res 1999, 18: 737-763

23. Dacey DM, Lee BB: The 'blue-on' opponent pathway in primate retina originates from a distinct bistratified ganglion cell type. Nature 1994, 367: 731735

24. Lee BB, Silveira LCL: Cone Opponency: An efficient way of transmitting chromatic information. In Human Color Vision. Edited by Kremers J, Baraas R, Marshall N. Springer Series in Vision Research 2016: 5, 105-132

25. Hood SM, Mollon JD, Purves L, Jordan G: Color discrimination in carriers of color deficiency. Vision Res 2006, 46: 2894-2900 
*26. Jacobs $\mathrm{GH}$ : Photopigments and the dimensionality of animal color vision. Neurosci Biobeh Rev 2018, 86:108-130

An excellent, comprehensive review about the link between photopigment complements and dimensionality of colour vision in the animal kingdom.

27. Bosten JM, Robinson JD, Jordan G, Mollon JD: Multidimensional scaling reveals a color dimension unique to 'color-deficient' observers. Curr Bio 2005, 15: R950-R952

28. Regan BC, Reffin JP, Mollon JD: Luminance noise and the rapid determination of discrimination ellipses in colour deficiency. Vision Res 1994 , 34: 1279-1299

29. Smeulders N, Campbell FW, Andrews PR: The role of delineations and spatial frequency in the perception of the colours of the spectrum. Vision Res 1994, 34: 927-936

30. Anstis S, Cavanagh P: A minimum motion technique for judging equiluminance. In Colour Vision: Physiology and Psychophysics. Edited by Mollon JD, Sharpe LT. Academic Press; 1983:155-166

31. Jordan G, Mollon JD: Eine evolutionäre Interpretation des menschlichen Farbensehens. Die Farbe 1988: 35/36, 139-170 
Fig 1. Retinal structures for tetrachromacy. (a) Relative sensitivities for the three normal cone photopigment S, M, L and two hybrid photopigments L' and M'. (b) Schematic representation of a foveal cone mosaic showing $S, M, L$ and $L$ ' cones in blue, green, red and orange respectively. Cone inputs into three possible circular centre-surround receptive fields of midget ganglion cells are superimposed on the cone mosaic. For information see text. 
(a)

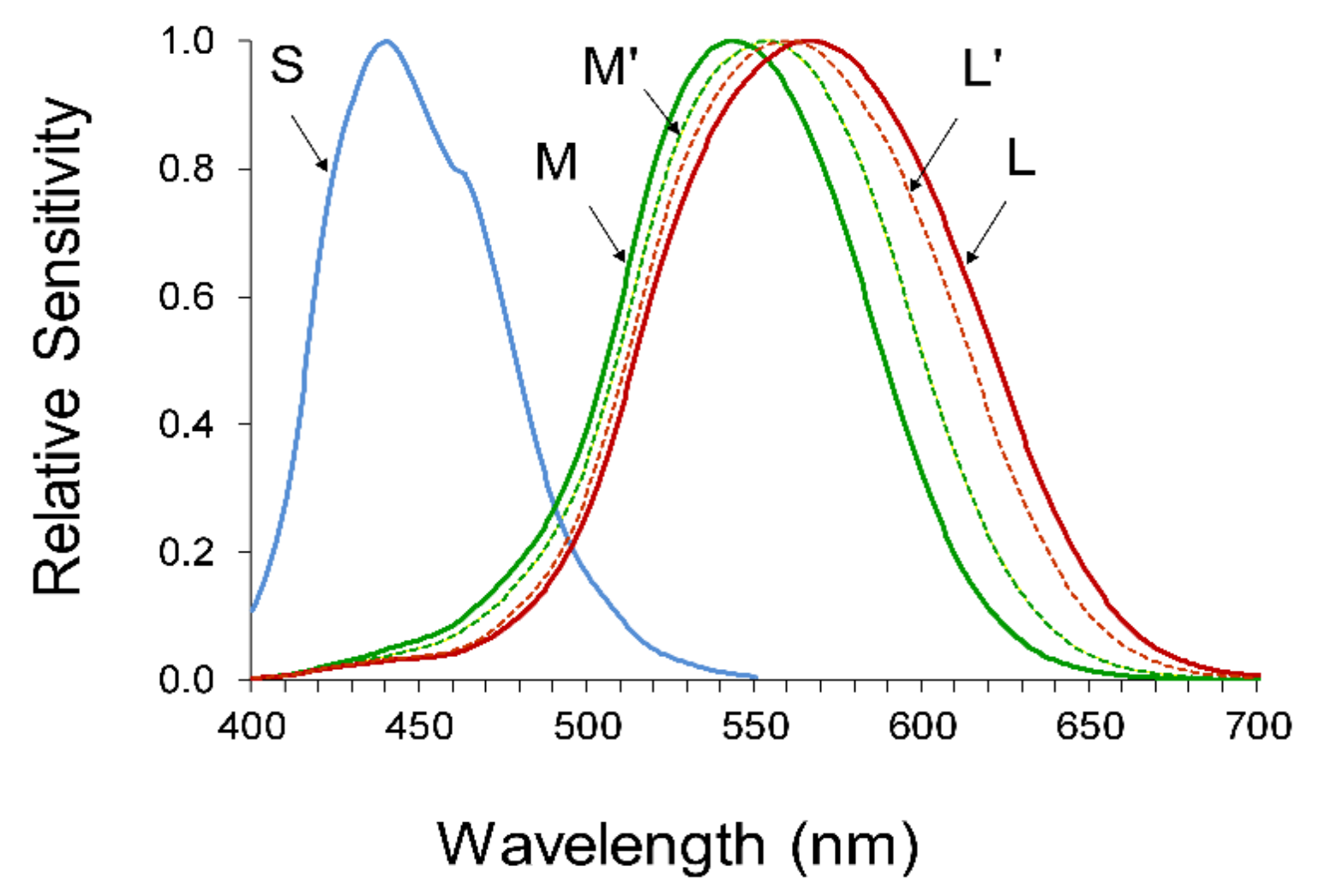

(b)

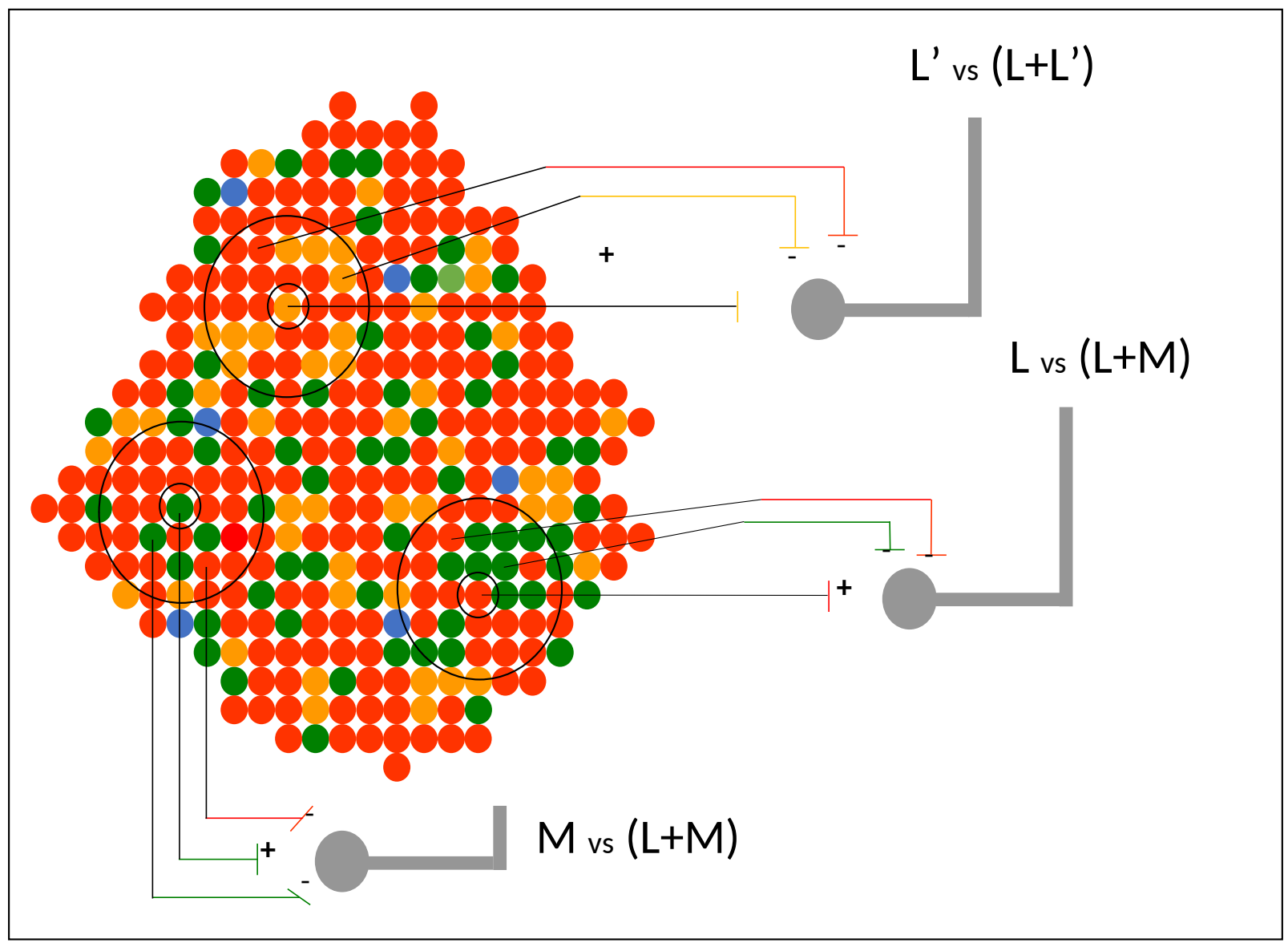


Tetrachromacy: The mysterious case of extra-ordinary color vision Gabriele Jordan and John Mollon

Conflict of interest statement

Nothing declared 